\title{
MEAN NUMBER OF REAL ZEROS OF A RANDOM TRIGONOMETRIC POLYNOMIAL. III
}

\author{
J. ERNEST WILKINS, JR. and SHANTAY A. SOUTER \\ Clark Atlanta University \\ Department of Mathematical Sciences \\ Atlanta, GA 30314 USA
}

(Received August, 1994; Revised March, 1995)

\begin{abstract}
If $a_{1}, a_{2}, \ldots, a_{n}$ are independent, normally distributed random variables with mean 0 and variance 1 , and if $\nu_{n}$ is the mean number of zeros on the interval $(0,2 \pi)$ of the trigonometric polynomial $a_{1} \cos x+2^{1 / 2} a_{2} \cos 2 x+\ldots n^{1 / 2} a_{n} \cos n x$, then $\nu_{n}=2^{-1 / 2}\left\{(2 n+1)+D_{1}+(2 n+1)^{-1} D_{2}+(2 n+1)^{-2} D_{3}\right\}+O\{(2 n+$ 1) $\left.{ }^{-3}\right\}$, in which $D_{1}=-0.378124, D_{2}=-\frac{1}{2}, D_{3}=0.5523$. After tabulation of $5 D$ values of $\nu_{n}$ when $n=1(1) 40$, we find that the approximate formula for $\nu_{n}$, obtained from the above result when the error term is neglected, produces $5 D$ values that are in error by at most $10^{-5}$ when $n \geq 8$, and by only about $0.1 \%$ when $n=2$.
\end{abstract}

Key words: Random Trigonometric Polynomials, Real Zeros.

AMS (MOS) subject classifications: 60G99.

\section{Introduction}

Suppose that $n$ is an integer greater than 1 , that $a_{j}(j=1,2, \ldots, n)$ are independent, normally distributed random variables with mean 0 and variance 1 , that $p$ is a real number greater than $-\frac{1}{2}$, and that $\nu_{n p}$ is the mean value of the number of zeros on the interval $(0,2 \pi)$ of the random trigonometric polynomial

$$
\sum_{j=1}^{n} j^{p} a_{j} \cos j x
$$

Das [4] has shown that, for large $n$,

$$
\nu_{n p}=2 \mu_{p} n+O\left(n^{1 / 2}\right), \mu_{p}=\{(2 p+1) /(2 p+3)\}^{1 / 2} .
$$

The author ([6] when $p=0$ and [7] when $p$ is a positive integer) has exhibited constants $D_{0 p}=1$, $D_{1 p}, D_{2 p}$ and $D_{3 p}$ such that

$$
\nu_{n p}=(2 n+1) \mu_{p} \sum_{r=0}^{3}(2 n+1)^{-r} D_{r p}+O\left\{(2 n+1)^{-3}\right\} .
$$

It follows that the error term $O\left(n^{1 / 2}\right)$ in the Das result is actually $O(1)$ when $p$ is a nonnegative integer. In this paper we will prove that a relation of the form (1.3) is also valid when $p=\frac{1}{2}$. This proof emulates the analysis in [7], although that analysis actually fails when $p$ is not a positive integer. 
After a statement of the basic formulas on which our analysis rests, we devote Section 2 to the derivation of a series representation of $\nu_{n, 1 / 2}$ that converges when $n$ is sufficiently large. (Henceforth we will omit the subscript $\frac{1}{2}$.) Asymptotic representations of the first four coefficients in that series are derived in Section 3, and are used to deduce (1.3) when $p=\frac{1}{2}$. We tabulate in Section $45 D$ values of $\nu_{n}$ when $n=1(1) 40$. We find that the approximation to $\nu_{n}$, obtained from (1.3) when the $O\left\{(2 n+1)^{-3}\right\}$ term is neglected, produces $5 D$ values that differ from the tabulated values by at most $10^{-5}$ when $n \geq 8$ and by only about $0.1 \%$ when $n=2$. In Section 5 we show that the series representation of $\nu_{n}$, derived in Section 2, actually converges when $n \geq 2$.

\section{Preliminary Analysis}

It is a consequence of the basic formulas in [7] (that were copied from [3, p. 285] or [2, p. 107]) that, when $n \geq 2$,

in which

$$
\nu_{n}=4 \pi^{-1} \int_{0}^{\pi / 2} F_{n}(x) d x
$$

$$
\begin{aligned}
F_{n}(x) & =A_{n}^{-1}\left(A_{n} C_{n}-B_{n}^{2}\right)^{1 / 2} \\
A_{n}=\sum_{j=1}^{n} j \cos ^{2} j x, \quad B_{n} & =\sum_{j=1}^{n} j^{2} \sin j x \cos j x, \quad C_{n}=\sum_{j=1}^{n} j^{3} \sin ^{2} j x .
\end{aligned}
$$

We will need the explicit representations of $A_{n}, B_{n}$ and $C_{n}$ stated in the following lemma.

Lemma 1: It is true that

$$
\begin{gathered}
8 A_{n}=(2 n+1)^{2} g_{0}(z)+(2 n+1) g_{1}+g_{2}, \\
16 B_{n}=(2 n+1)^{3} h_{0}(z)+(2 n+1)^{2} h_{1}+(2 n+1) h_{2}+h_{3}, \\
32 C_{n}=(2 n+1)^{4} k_{0}(z)+(2 n+1)^{3} k_{1}+(2 n+1)^{2} k_{2}+(2 n+1) k_{3}+k_{4},
\end{gathered}
$$

in which

$$
\begin{gathered}
z=(2 n+1) x, f(x)=\csc x-x^{-1}, \varphi(x)=f^{2}(x)+2 x^{-1} f(x)=c s c^{2} x-x^{-2}, \\
g_{0}(z)=\left(\frac{1}{2}\right)+z^{-1} \sin z-z^{-2}(1-\cos z), g_{1}=f(x) \sin z \\
g_{2}=-\left\{\left(\frac{1}{2}\right)+\varphi(x)+f^{\prime}(x) \cos z\right\} \\
h_{0}(z)=-g_{0}^{\prime}(z)=-z^{-1} \cos z+2 z^{-2} \sin z-2 z^{-3}(1-\cos z), \\
h_{1}=-f(x) \cos z, h_{2}=-2 f^{\prime}(x) \sin z, h_{3}=\varphi^{\prime}(x)+f^{\prime \prime}(x) \cos z, \\
k_{0}(z)=\left(\frac{1}{4}\right)-z^{-1} \sin z-3 z^{-2} \cos z+6 z^{-3} \sin z-6 z^{-4}(1-\cos z), \\
k_{1}=-f(x) \sin z, k_{2}=3 f^{\prime}(x) \cos z-\left(\frac{1}{2}\right), k_{3}=3 f^{\prime \prime}(x) \sin z
\end{gathered}
$$




$$
k_{4}=\left(\frac{1}{4}\right)-\varphi^{\prime \prime}(x)-f^{\prime \prime \prime}(x) \cos z
$$

With the help of a known trigonometric summation [5, p. 133, Eq. (31)], we see that

$$
\begin{gathered}
8 A_{n}=4 \sum_{j=1}^{n} j+4 \sum_{j=1}^{n} j \cos 2 j x \\
=2 n(n+1)+2\{(n+1) \sin z-\sin x\} \csc x+\{\cos (z+x)-\cos 2 x\} \csc ^{2} x .
\end{gathered}
$$

A little trigonometric and algebraic manipulation then suffices to establish (2.4), (2.7), (2.8), and (2.9). The remaining results of the lemma are consequences of these and the inferences from (2.3) that

$$
2 B_{n}=-d A_{n} / d x, 2 C_{n}=\sum_{j=1}^{n} j^{3}-d B_{n} / d x=\{n(n+1) / 2\}^{2}-d B_{n} / d x .
$$

We calculate the first factor $A_{n}^{-1}$ in $(2.2)$ in the following lemma.

Lemma 2: There is a positive integer $n_{0}$ such that, if $0 \leq x \leq \pi / 2$ and $n \geq n_{0}$,

$$
\left(8 A_{n}\right)^{-1}=(2 n+1)^{-2} g_{0}^{-1} \sum_{r=0}^{\infty}(2 n+1)^{-r} b_{r},
$$

in which $b_{0}=1, \quad b_{1}=-g_{1} / g_{0}, b_{2}=b_{1}^{2}-\left(g_{2} / g_{0}\right), b_{3}=\left(2 g_{1} g_{2} / g_{0}^{2}\right)+b_{1}^{3}, \ldots$ The series converges absolutely and uniformly when $0 \leq x \leq \pi / 2$ and $n \geq n_{0}$.

It is a consequence of $(2.8)$ that

$$
g_{0}=\int_{0}^{1} t(1+\cos z t) d t .
$$

Therefore, $g_{0}>0$ when $0 \leq z<+\infty$, and $\lim _{z \rightarrow \infty} g_{0}=\frac{1}{2}$, so that both $g_{0}$ and $g_{0}^{-1}$ are bounded functions of $z$. Because the functions $g_{1}$ and $g_{2}$, defined in (2.8) and (2.9), are obviously bounded, uniformly in $x$ and $n$ when $0 \leq x \leq \pi / 2$ and $n \geq 2$, it follows that there exists a positive integer $n_{0}$ so large that

$$
(2 n+1)^{-1}\left|g_{1} / g_{0}\right|+(2 n+1)^{-2}\left|g_{2} / g_{0}\right|<0.65
$$

when $n \geq n_{0}$ and $0 \leq x \leq \pi / 2$. The expression (2.4) can be inverted for such values of $x$ and $n$; this process yields (2.15), in which the first four coefficients are those specified in the lemma.

A straightforward calculation based on (2.4), (2.5) and (2.6), proves the following lemma.

Lemma 3: It is true that

$$
256\left(A_{n} C_{n}-B_{n}^{2}\right)=(2 n+1)^{6} \sum_{r=0}^{6}(2 n+1)^{-r} f_{r}
$$

in which

$$
f_{r}=\sum_{m=0}^{r}\left(g_{m} k_{r-m}-h_{m} h_{r-m}\right) \quad(r=1,2, \ldots, 6),
$$

and $g_{m}=0$ if $m>2, h_{m}=0$ if $m>3$ and $k_{m}=0$ if $m>4$.

We calculate the second factor $\left(A_{n} C_{n}-B_{n}^{2}\right)^{1 / 2}$ of (2.2) in the following lemma.

Lemma 4: The integer $n_{0}$ of Lemma 2 may be chosen so that, if $0 \leq x \leq \pi / 2$ and $n \geq n_{0}$, 


$$
16\left(A_{n} C_{n}-B_{n}^{2}\right)^{1 / 2}=(2 n+1)^{3} f_{0}^{1 / 2} \sum_{r=0}^{\infty}(2 n+1)^{-r} c_{r}
$$

in which $c_{0}=1, \quad c_{1}=f_{1} /\left(2 f_{0}\right), \quad c_{2}=f_{2} /\left(2 f_{0}\right)-f_{1}^{2} /\left(8 f_{0}^{2}\right), \quad c_{3}=f_{3} /\left(2 f_{0}\right)-f_{1} f_{2} /\left(4 f_{0}^{2}\right)+f_{1}^{3} /$ $\left(16 f_{0}^{3}\right), \ldots$ The series $(2.20)$ converges absolutely and uniformly when $0 \leq x \leq \pi / 2$ and $n \geq n_{0}$.

We infer from (2.10) and (2.12) that

$$
h_{0}=\int_{0}^{1} t^{2} \sin z t d t, k_{0}=\int_{0}^{1} t^{3}(1-\cos z t) d t,
$$

so that (2.16) and the Schwartz inequality imply that $f_{0}=g_{0} k_{0}-h_{0}^{2}>0$ when $0<z<+\infty$. Moreover, $f_{0}=z^{2} / 48+O\left(z^{4}\right)$ for small $z$, and $f_{0}=1 / 8+O\left(z^{-1}\right)$ for large $z$.

Because $f(x)$ is an odd analytic function of $x$ when $|x|<\pi$, and because $0 \leq x=z /(2 n+1) \leq z / 5$ when $n \geq 2$, it follows from (2.21) and (2.11) that $h_{r}=O(z)(r=0,1$, $2, \overline{3})$, uniformly in $\bar{x}$ and $n$, and from (2.21), (2.13) and (2.14) that $k_{r}=O\left(z^{2}\right) \quad(r=0,1,2,3,4)$, uniformly in $x$ and $n$. It is now a consequence of (2.19) and the earlier observation that $g_{r}=O(1)(r=0,1,2)$, uniformly in $x$ and $n$, that $f_{r}=O\left(z^{2}\right) \quad(r=1,2,3,4,5,6)$, uniformly in $x$ and $n$. Because it is obvious that $h_{r}=O(1)$ and $k_{r}=O(1)$, uniformly in $x$ and $n$, we conclude that $f_{r} / f_{0}=O(1)$, uniformly in $x$ and $n$ (whether or not $z$ is small). Hence we can choose $n_{0}$ so large that (2.17) holds, and

$$
\sum_{r=1}^{6}(2 n+1)^{-r}\left|f_{r} / f_{0}\right|<0.92
$$

when $n \geq n_{0}$ and $0 \leq x \leq \pi / 2$. For such values of $x$ and $n$ the square root of the expression (2.18) can be written in the form (2.20), the first four coefficients of which are those specified in the lemma.

We will show in Section 5 that the inequalities (2.17) and (2.22) hold when $n \geq 2$. Therefore, Lemmas 2 and 4 (also Lemmas 5 and 6 below) are valid when $n_{0}=2$.

If we use (2.2) and Lemmas 2 and 4, we obtain the following lemma.

Lemma 5: It is true when $0 \leq x \leq \pi / 2$ and $n \geq n_{0}$ that

$$
2 F_{n}(x)=(2 n+1) G(z) \sum_{r=0}^{\infty}(2 n+1)^{-r} u_{r}
$$

in which

$$
G(z)=f_{0}^{1 / 2}(z) / g_{0}(z), u_{r}=\sum_{m=0}^{r} b_{m} c_{r-m} .
$$

Moreover, the series (2.23) converges absolutely and uniformly in $x$ and $n$.

The final lemma in this section is a consequence of (2.1) and Lemma 5.

Lemma 6: It is true when $n \geq n_{0}$ that

in which

$$
\nu_{n}=(2 n+1) \sum_{r=0}^{\infty}(2 n+1)^{-r} v_{r}
$$

$$
v_{r}=2 \pi^{-1} \int_{0}^{\pi / 2} G(z) u_{r} d x
$$


Moreover, the series (2.25) converges' absolutely and uniformly when $n \geq n_{0}$.

\section{Proof of (1.3) when $p=\frac{1}{2}$}

In the next four lemmas we will exhibit constants $S_{r m}(0 \leq m \leq 3-r, r=0,1,2,3)$ and $S_{r}$ $(r=0,1,2,3)$ such that

$$
2^{1 / 2} v_{r}=\sum_{m=0}^{3-r}(2 n+1)^{-m} S_{r m}+(-1)^{n}(2 n+1)^{r-3} S_{r}+O\left\{(2 n+1)^{r-4}\right\}
$$

when $r=0,1,2,3$. In the proofs of these lemmas, it will be convenient to use $T_{q}(z)$ as a generic symbol for a trigonometric sine polynomial of degree $q$, not necessarily the same at each occurrence.

Lemma 7: Equation (3.1) is true when $r=0$ if

$$
\begin{gathered}
S_{00}=1, S_{01}=(2 / \pi) \int_{0}^{\infty}\left\{2^{1 / 2} G(z)-1\right\} d z \\
S_{02}=1 / \pi^{2}, S_{03}=0, S_{0}=-32 / \pi^{3} .
\end{gathered}
$$

It follows from $(2.19),(2.8),(2.10)$ and $(2.12)$ that

$$
\begin{gathered}
8 f_{0}=1-2 z^{-1} \sin z-10 z^{-2}(1+\cos z)+32 z^{-3} \sin z-8 z^{-4}(1-\cos z)^{2} \\
-32 z^{-5}(1-\cos z) \sin z+16 z^{-6}(1-\cos z)^{2}
\end{gathered}
$$

For sufficiently large $z$ we conclude that

$$
\begin{gathered}
\left(8 f_{0}\right)^{-1}=1+2 z^{-1} \sin z+2 z^{-2}(6+5 \cos z-\cos 2 z)+z^{-3} T_{3}(z)+O\left(z^{-4}\right) \\
\left(8 f_{0}\right)^{1 / 2}=1-z^{-1} \sin z-(4 z)^{-2}(21+20 \cos z-\cos 2 z) \\
+z^{-3} T_{3}(z)+O\left(z^{-4}\right) .
\end{gathered}
$$

Because it follows from (2.8) that

$$
\left(2 g_{0}\right)^{-1}=1-2 z^{-1} \sin z+2 z^{-2}(2-\cos z-\cos 2 z)+z^{-3} T_{3}(z)+O\left(z^{-4}\right),
$$

we infer from (2.24) and (3.6) that

$$
\begin{gathered}
2^{1 / 2} G(z)=1-3 z^{-1} \sin z-(4 z)^{-2}(1+28 \cos z+11 \cos 2 z) \\
+z^{-3} T_{3}(z)+O\left(z^{-4}\right)
\end{gathered}
$$

If we define $\lambda$ to be $(2 n+1) \pi / 2$, it follows from $(2.26)$ that

$$
2^{1 / 2} v_{0}=1+\lambda^{-1} \int_{0}^{\infty}\left\{2^{1 / 2} G(z)-1\right\} d z-\lambda^{-1} \int_{\lambda}^{\infty}\left\{2^{1 / 2} G(z)-1\right\} d z
$$

the improper integrals exist by virtue of (3.8). Repeated integration by parts of the last term in 
(3.9) now shows, with the help of (3.8), that the lemma is true.

Lemma 8: Equation (3.1) is true when $r=1$ if

in which

$$
\begin{gathered}
S_{10}=0, S_{11}=-(5 / \pi) \int_{0}^{\pi / 2} x^{-1} f(x) d x, S_{1}=-24 / \pi^{3}, \\
S_{12}=-(3 \pi)^{-1} \int_{0}^{\infty} z\left\{2^{1 / 2} H(z)-3 \sin z-(2 z)^{-1}(5+11 \cos 2 z)\right\} d z
\end{gathered}
$$

$$
H(z)=\left[\left\{\left(2 f_{0}\right)^{-1}\left(g_{0}-k_{0}\right)+g_{0}^{-1}\right\} \sin z-f_{0}^{-1} h_{0} \cos z\right] G(z) .
$$

It follows from (2.19), (2.8), (2.11) and (2.13) that

$$
f_{1}=-\left\{\left(g_{0}-k_{0}\right) \sin z-2 h_{0} \cos z\right\} f(x),
$$

and then from (2.8), (2.10) and (2.12) that

$$
\begin{gathered}
f_{1}=-\left\{4^{-1} \sin z+2 z^{-1}-z^{-2} \sin z-z^{-3}(5-4 \cos z-\cos 2 z)\right. \\
\left.+6 z^{-4}(1-\cos z) \sin z\right\} f(x) .
\end{gathered}
$$

We can now deduce from (2.24), Lemmas 2 and 4 , and (3.13) that

$$
G(z) u_{1}=-f(x) H(z),
$$

in which $H(z)$ is the function defined in (3.12). With the help of (3.5), (3.7), (3.8) and the definitions (2.8), (2.10) and (2.12), it is easy to see that, for large $z$,

$$
H(z)=H^{*}(z)+O\left(z^{-3}\right)
$$

in which

$$
\begin{aligned}
& 2^{1 / 2} H^{*}(z)=3 \sin z+(2 z)^{-1}(5+11 \cos 2 z) \\
& +8^{-1} z^{-2}(151 \sin z-60 \sin 2 z-69 \sin 3 z)
\end{aligned}
$$

Moreover, $H(z)=O(1)$ and $H^{*}(z)=O\left(z^{-1}\right)$ for small $z$. Hence (3.16) is true for all positive $z$. It now follows from (2.26) and (3.15) that

$$
-\pi v_{1} / 2=I_{1}+I_{2}+I_{3}
$$

in which

$$
\begin{gathered}
I_{1}=\int_{0}^{\pi / 2}\{f(x)-(x / 6)\}\left\{H(z)-H^{*}(z)\right\} d x \\
I_{2}=\int_{0}^{\pi / 2}(x / 6)\left\{H(z)-H^{*}(z)\right\} d x
\end{gathered}
$$




$$
I_{3}=\int_{0}^{\pi / 2} f(x) H^{*}(z) d x
$$

We recall the identity [6, Lemma 2],

$$
\begin{aligned}
f(x) & =\sum_{m=1}^{\infty}(-1)^{m-1}\left(2^{2 m}-2\right) \beta_{2 m} x^{2 m-1} /(2 m) ! \\
& =(x / 6)+\left(7 x^{3} / 360\right)+\left(31 x^{5} / 15120\right)+\ldots,
\end{aligned}
$$

in which $\beta_{2 m}$ is the Bernoulli number of order $2 m$ [1, p. 804]. It is known [1, pp. 75, 805] that the power series in (3.22) converges when $|x|<\pi$, and that all of its nonzero coefficients are positive. We conclude from (3.22), (3.16), and (3.19) that

$$
I_{1}=\int_{0}^{\pi / 2} O\left(x^{3}\right) O\left(z^{-3}\right) d x=O\left\{(2 n+1)^{-3}\right\}
$$

Similarly, we deduce from (3.16) that

$$
\begin{gathered}
6 I_{2}=(2 n+1)^{-2} \int_{0}^{\lambda} z\left\{H(z)-H^{*}(z)\right\} d z \\
=(2 n+1)^{-2} \int_{0}^{\infty} z\left\{H(z)-H^{*}(z)\right\} d z-(2 n+1)^{-2} \int_{\lambda}^{\infty} z O\left(z^{-3}\right) d z \\
6 \cdot 2^{1 / 2} I_{2}=(2 n+1)^{-2}\left[\int _ { 0 } ^ { \infty } z \left\{2^{1 / 2} H(z)-3 \sin z\right.\right. \\
\left.\left.-(2 z)^{-1}(5+11 \cos 2 z)\right\} d z-11 \pi / 8\right]+O\left\{(2 n+1)^{-3}\right\} .
\end{gathered}
$$

In order to evaluate the integral $I_{3}$, we use the results [7, Eqs. (3.15)],

$$
\begin{gathered}
\int_{0}^{\pi / 2} f(x) \sin z d x=(2 n+1)^{-2}\left(4 / \pi^{2}\right)(-1)^{n}+O\left\{(2 n+1)^{-3}\right\} \\
\int_{0}^{\pi / 2} f(x) z^{-1} d x=(2 n+1)^{-1} \int_{0}^{\pi / 2} f(x) x^{-1} d x \\
\int_{0}^{\pi / 2} f(x) z^{-1} \cos 2 z d x=O\left\{(2 n+1)^{-3}\right\} \\
\int_{0}^{\pi / 2} f(x) z^{-2} \sin q z d x=(2 n+1)^{-2}(\pi / 12)+O\left\{(2 n+1)^{-3}\right\}
\end{gathered}
$$

when $q>0$. Therefore,

$$
2^{1 / 2} I_{3}=(5 / 2)(2 n+1)^{-1} \int_{0}^{\pi / 2} f(x) x^{-1} d x+\left(12 / \pi^{2}\right)(2 n+1)^{-2}(-1)^{n}
$$




$$
+(11 \pi / 48)(2 n+1)^{-2}+O\left\{(2 n+1)^{-3}\right\} .
$$

We now combine (3.23), (3.24) and (3.25) with (3.18) to conclude that Lemma 8 is true.

Lemma 9: Equation (3.1) is true when $r=2$ if

$$
\begin{gathered}
S_{20}=(2 / \pi) \int_{0}^{\pi / 2} \varphi(x) d x-(5 / 2 \pi) \int_{0}^{\pi / 2} f^{2}(x) d x-\left(\frac{1}{2}\right), \\
S_{21}=(1 / 3 \pi) \int_{0}^{\infty}\left\{2^{1 / 2} J(z)+1-7 \cos z\right\} d z, S_{2}=56 / \pi^{3},
\end{gathered}
$$

in which

$$
\begin{gathered}
J(z)=\left[(1-\cos z)\left\{\left(2 f_{0}\right)^{-1}\left(k_{0}-3 g_{0}\right)-g_{0}^{-1}\right\}+2 f_{0}^{-1} h_{0} \sin z\right. \\
\left.+6 g_{0}^{-1}-3 f_{0}^{-1} k_{0}\right] G(z) .
\end{gathered}
$$

It follow from $(2.19),(2.8),(2.9),(2.11)$ and $(2.13)$ that

$$
f_{2}=\left\{\left(3 g_{0}-k_{0}\right) \cos z+4 h_{0} \sin z\right\} f^{\prime}(x)-f^{2}(x)-k_{0} \varphi(x)-\left(g_{0}+k_{0}\right) / 2,
$$

and then from (2.24), Lemmas 2 and $4,(3.13)$ and (3.29) that

$$
G u_{2}=f^{\prime}(0) J(z)+\left\{f^{\prime}(x)-f^{\prime}(0)\left\{K(z)-f^{2}(x) L(z)+\{\varphi(x)-\varphi(0)\} M(z),\right.\right.
$$

in which $J(z)$ is defined in $(3.28)$ and

$$
\begin{gathered}
K(z)=\left[\left\{\left(2 f_{0}\right)^{-1}\left(3 g_{0}-k_{0}\right)+g_{0}^{-1}\right\} \cos z+2 h_{0} f_{0}^{-1} \sin z\right] G(z), \\
L(z)=\left[( 1 - \operatorname { c o s } 2 z ) \left\{\left(4 f_{0}\right)^{-2}\left[\left(g_{0}-k_{0}\right)^{2}-4 h_{0}^{2}\right]-\left(4 f_{0} g_{0}\right)^{-1}\left(g_{0}-k_{0}\right)\right.\right. \\
\left.-\left(2 g_{0}^{2}\right)^{-1}\right\}+\left\{g_{0}^{-1}-\left(2 f_{0}\right)^{-1}\left(g_{0}-k_{0}\right)\right\}\left(2 f_{0}\right)^{-1} h_{0} \sin 2 z \\
\left.+\left(2 f_{0}^{2}\right)^{-1}\left(h_{0}^{2}+f_{0}\right)\right] G(z), \\
M(z)=\left\{g_{0}^{-1}-\left(2 f_{0}\right)^{-1} k_{0}\right\} G(z) .
\end{gathered}
$$

With the help of (3.5), (3.7), (3.8) and the definitions (2.8), (2.10) and (2.12), it is easy to see that, for large $z$,

$$
2^{1 / 2} J(z)=-1+7 \cos z-z^{-1}(31+15 \cos z) \sin z+O\left(z^{-2}\right) .
$$

In fact, (3.34) is valid for all positive $z$ because $J(z)$ and $z^{-1} \sin z$ are bounded. It then follows that

$$
\int_{0}^{\pi / 2} 2^{1 / 2} J(z) d x=-(\pi / 2)+\left[7(-1)^{n}+\int_{0}^{\infty}\left\{2^{1 / 2} J(z)+1-7 \cos z\right\} d z\right.
$$




$$
\left.-\int_{\lambda}^{\infty}\left\{2^{1 / 2} J(z)+1-7 \cos z\right\} d z\right](2 n+1)^{-1} .
$$

In view of (3.34) the two improper integrals converge, and the last integral in (3.35) is $O\left\{(2 n+1)^{-1}\right\}$. In a similar manner, we find that

$$
\begin{gathered}
2^{1 / 2} K(z)=7 \cos z-15 z^{-1} \sin z \cos z+O\left(z^{-2}\right), \\
2^{1 / 2} L(z)=(5+11 \cos 2 z) / 4+z^{-1}(111 \sin z-69 \sin 3 z) / 8+O\left(z^{-2}\right), \\
2^{1 / 2} M(z)=1-2 z^{-1} \sin z+O\left(z^{-2}\right) \\
2^{1 / 2} \int_{0}^{\pi / 2}\left\{f^{\prime}(x)-f^{\prime}(0)\right\} K(z) d x=7\left\{(2 / \pi)^{2}-(1 / 6)\right\}(-1)^{n}(2 n+1)^{-1} \\
+O\left\{(2 n+1)^{-2}\right\} \\
2^{1 / 2} \int_{0}^{\pi / 2} f^{2}(x) L(z) d x=(5 / 4) \int_{0}^{\pi / 2} f^{2}(x) d x+O\left\{(2 n+1)^{-2}\right\}, \\
\int_{0}^{\pi / 2}\{\varphi(x)-\varphi(0)\} M(z) d x=\int_{0}^{\pi / 2} \varphi(x) d x-(\pi / 6)+O\left\{(2 n+1)^{-2}\right\} .
\end{gathered}
$$

It now follows from (2.26), (3.30), (3.35), (3.39), (3.40) and (3.41) that Lemma 9 is true.

Lemma 10: Equation (3.1) is true when $r=3$ if $S_{30}=0, S_{3}=0$.

It follows from (2.19) and (2.8) through (2.13) that

$$
f_{3}=\left(3 g_{0} \sin z-2 h_{0} \cos z\right) f^{\prime \prime}(x)+f(x) \varphi(x) \sin z-2 h_{0} \varphi^{\prime}(x)
$$

and then from (2.24), Lemmas 2 and $4,(3.13),(3.29)$ and (3.42) that

$$
\begin{aligned}
2^{1 / 2} G u_{3}=f^{\prime \prime}(x) N(z)+ & f(x) \varphi(x) P(z)+\varphi^{\prime}(x) Q(z)+f(x)\left\{f^{\prime}(x)-f^{\prime}(0)\right\} R(z) \\
& +f^{3}(x) U(z)+f(x) V(z)
\end{aligned}
$$

in which $N, P, Q, R, U$ and $V$ are certain explicitly definable functions of $z$ concerning which we need to know first that, for large $z$,

$$
\begin{gathered}
N(z)=6 \sin z+O\left(z^{-1}\right), P(z)=-5 \sin z+O\left(z^{-1}\right), Q(z)=O\left(z^{-1}\right) \\
R(z)=-7.5 \sin 2 z+(4 z)^{-1}(193 \cos z-97 \cos 3 z)+O\left(z^{-2}\right) \\
U(z)=8^{-1}(23 \sin 3 z-37 \sin z)-(16 z)^{-1}(95+132 \cos 2 z \\
-99 \cos 4 z)+O\left(z^{-2}\right) \\
V(z)=-5 \sin z-(35 / 6) \sin 2 z+O\left(z^{-1}\right) .
\end{gathered}
$$


We also need to know that, for small positive $z$, the functions $N(z), P(z), Q(z)$, and $V(z)$ are bounded (they actually have limits as $z \rightarrow 0^{+}$), and the functions $R(z)$ and $U(z)$ are $O\left(z^{-2}\right)$. Hence equations (3.44) through (3.47) are valid for all positive $z$. Each of the integrals with respect to $x$ on the interval $(0, \pi / 2)$ of each of the six terms on the right hand side of (3.43) is, therefore, $O\left\{(2 n+1)^{-1}\right\}$. In view of $(2.26)$, this remark suffices to prove Lemma 10 .

Our principal result, stated in the following theorem, is an immediate consequence of Lemmas 6 through 10 and the observation that $S_{0}+S_{1}+S_{2}+S_{3}=0$.

Theorem: When $p=\frac{1}{2}$ and $n \geq n_{0}$, the mean value of the number of zeros on the interval $(0,2 \pi)$ of the random trigonometric polynomial (1.1) is

$$
\nu_{n}=(2 n+1) 2^{-1 / 2} \sum_{r=0}^{3}(2 n+1)^{-r} D_{r}+O\left\{(2 n+1)^{-3}\right\}
$$

in which

$$
D_{r}=\sum_{m=0}^{r} S_{r-m, m} \quad(r=0,1,2,3)
$$

If we use the explicit formulas for $S_{r m}$ furnished in Lemmas 7 through 10, it follows that

$$
\begin{gathered}
D_{0}=1, D_{1}=(2 / \pi) \int_{0}^{\infty}\left\{2^{1 / 2} G(z)-1\right\} d z, D_{2}=-\frac{1}{2} \\
D_{3}=(1 / 3 \pi) \int_{0}^{\infty}\left\{2^{1 / 2} J(z)+1-7 \cos z\right\} d z \\
-(1 / 3 \pi) \int_{0}^{\infty} z\left\{2^{1 / 2} H(z)-3 \sin z-(2 z)^{-1}(5+11 \cos 2 z)\right\} d z
\end{gathered}
$$

(For the calculation of $D_{2}$, it is necessary to know [6, Eq. (3.56)] that $\int_{0}^{\pi / 2} \varphi(x) d x=2 / \pi$.)

\section{Numerical Results}

With the help of (3.8), (3.16), (3.17), and (3.34), we can transform the conditionally convergent integrals in (3.50) and (3.51) into absolutely convergent integrals, i.e.,

$$
\begin{gathered}
D_{1}=(2 / \pi) \int_{0}^{\infty}\left\{2^{1 / 2} G(z)-1+3 z^{-1} \sin z\right\} d z-3 \\
D_{3}=(1 / 3 \pi) \int_{0}^{\infty}\left[2^{1 / 2}\{J(z)-z H(z)\}+3 z \sin z+2^{-1}(7-14 \cos z+11 \cos 2 z)\right. \\
\left.+(8 z)^{-1}(399 \sin z-69 \sin 3 z)\right\} d z-(55 / 8)
\end{gathered}
$$

We calculated the integrals in $(4.1)$ and $(4.2)$ over the interval $(0,25 \pi)$ by Simpson's rule. The integrals over the interval $(25 \pi, \infty)$ were calculated using the asymptotic relations, 


$$
\begin{gathered}
2^{1 / 2} G(z)-1+3 z^{-1} \sin z=-(2 z)^{-2}(1+28 \cos z+11 \cos 2 z) \\
+(2 z)^{-3}(19 \sin z+60 \sin 2 z+23 \sin 3 z)-2^{-6} z^{-4}(1063+2376 \cos z \\
+716 \cos 2 z-776 \cos 3 z-179 \cos 4 z)+z^{-5} T_{5}(z)+O\left(z^{-6}\right) \\
2^{1 / 2} H(z)-3 \sin z-(2 z)^{-1}(5+11 \cos 2 z) \\
-2^{-3} z^{-2}(151 \sin z-60 \sin 2 z-69 \sin 3 z) \\
=2^{-4} z^{-3}(207+1028 \cos z+612 \cos 2 z-388 \cos 3 z \\
-179 \cos 4 z)+z^{-4} T_{5}(z)+O\left(z^{-5}\right) \\
2^{1 / 2} J(z)+1-7 \cos z+(2 z)^{-1}(62 \sin z+15 \sin 2 z) \\
=2^{-3} z^{-2}(46-335 \cos z-478 \cos 2 z-97 \cos 3 z)+z^{-3} T_{4}(z)+O\left(z^{-4}\right)
\end{gathered}
$$

these relations contain one or two more terms than (3.8), (3.16) and (3.34). In this manner, we find that $D_{1}=-0.378124, D_{3}=0.5523$. Hence

$$
2^{1 / 2} \nu_{n}=(2 n+1)-0.378124-2^{-1}(2 n+1)^{-1}+0.5523(2 n+1)^{-2}+O\left\{(2 n+1)^{-3}\right\} .
$$

We have also used Simpson's rule to calculate numerical values of the integral (2.1) when $n=2(1) 40$. The results for $\nu_{n}$ are recorded to $5 D$ in Table 1 . (The tabulated value $\nu_{1}=2$ is obviously correct, but is not a consequence of (2.1) which is nugatory when $n=1$.) The approximation $2^{-1 / 2}\left(2 n+1+D_{1}\right)$ produces $5 D$ values that exceed the values in Table 1 by about $0.00756 \%$ when $n=40,0.0292 \%$ when $n=20,0.110 \%$ when $n=10$ and only $1.61 \%$ when $n=2$. The more accurate approximation $2^{-1 / 2}\left\{2 n+1+D_{1}-(4 n+2)^{-1}\right\}$ produces $5 D$ values that are less than the values in Table 1 by about $6 \times 10^{-5}$ when $n=40,23 \times 10^{-5}$ when $n=20$, $89 \times 10^{-5}$ when $n=10$, and only 0.0189 or $0.588 \%$ when $n=2$. The most accurate approximation $2^{-1 / 2}\left\{2 n+1+D_{1}-(4 n+2)^{-1}+0.5523(2 n+1)^{-2}\right\}$ produces $5 D$ values that agree with those in Table 1 when $n \geq 8$, except for a discrepancy of one unit in the last decimal place when $n=14$. Moreover, it produces a $5 D$ value when $n=2$ that is less than the Table 1 value by only 0.00328 , or $0.102 \%$. 


\begin{tabular}{|l|l|l|l|}
\hline$n$ & $\nu_{n}$ & $n$ & $\nu_{n}$ \\
\hline & & & \\
1 & 2 & 21 & 30.13021 \\
2 & 3.21635 & 22 & 31.54477 \\
3 & 4.64048 & 23 & 32.95930 \\
4 & 6.06232 & 24 & 34.37381 \\
5 & 7.48196 & 25 & 35.78829 \\
6 & 8.90016 & 26 & 37.20275 \\
7 & 10.31741 & 27 & 38.61720 \\
8 & 11.73400 & 28 & 40.03163 \\
9 & 13.15013 & 29 & 41.44605 \\
10 & 14.56592 & 30 & 42.86045 \\
11 & 15.98145 & 31 & 44.27484 \\
12 & 17.39678 & 32 & 45.68922 \\
13 & 18.81195 & 33 & 47.10359 \\
14 & 20.22699 & 34 & 48.51795 \\
15 & 21.64194 & 35 & 49.93231 \\
16 & 23.05679 & 36 & 51.34665 \\
17 & 24.47158 & 37 & 52.76099 \\
18 & 25.88631 & 38 & 54.17532 \\
19 & 27.30098 & 39 & 55.58965 \\
20 & 28.71561 & 40 & 57.00397 \\
\hline
\end{tabular}

Table 1. Values of the mean number of zeros of the random trigonometric polynomial (1.1) when $p=\frac{1}{2}$.

\section{The Integer $n_{0}$}

Although it is not logically necessary to know a specific value for the integer $n_{0}$ in the theorem and Lemmas 2, 4, 5 and 6 , it is interesting to observe that $n_{0}$ can actually be chosen as small as 2 . We begin the proof of this assertion with the following lemma.

Lemma 11: It is true that $g_{0}(z)>0.206715$ when $0 \leq z<\infty$.

It follows from (2.16) that

and from (2.10) that

$$
g_{0}^{\prime}(z)=-\int_{0}^{1} t^{2} \sin z t d t<0 \text { when } 0<z \leq \pi
$$

$$
g_{0}^{\prime}(z)>0 \text { when } 3 \pi / 2 \leq z \leq 2 \pi .
$$

If we define $W(z)$ to be $z^{4} g_{0}^{\prime \prime}(z)$, we infer from (5.1) and (2.10) that

$$
W(z)=-\int_{0}^{z} u^{3} \cos u d u=-z^{3} \sin z-3 z^{2} \cos z+6 z \sin z-6(1-\cos z) .
$$

Therefore, if $\pi \leq z<3 \pi / 2, W^{\prime}(z)=-z^{3} \cos z>0$, so that $W(z) \geq W(\pi)=3\left(\pi^{2}-4\right)>0$. Hence $g_{0}^{\prime \prime}(z)>0$ when $\pi \leq z \leq 3 \pi / 2$, and there exists a unique $z_{1}$ such that $\pi<z_{1}<3 \pi / 2, g_{0}^{\prime}\left(z_{1}\right)=0$. 
By virtue of (5.1) and (5.2), the minimum value of $g_{0}(z)$ on the interval $(0,2 \pi)$ is $g_{0}\left(z_{1}\right)$.

We use Newton's method, starting with the value $z=4 \pi / 3$, to locate the point $z_{1}$ with sufficient accuracy to determine that $g_{0}\left(z_{1}\right)>0.206715$, so that $g_{0}(z)>0.206715$ when $0 \leq z \leq 2 \pi$. If $z \geq 2 \pi$, we conclude from $(2.8)$ that $g_{0}(z) \geq 2^{-1}-z^{-1}-2 z^{-2} \geq 2^{-1}-(2 \pi)^{-1}-2(2 \pi)^{-2}>$ 0.290184 . The proof of the lemma is now complete.

Lemma 12: It is true when $0 \leq x \leq \pi / 2$ and $n \geq 2$ that

$$
\left|g_{1}\right| \leq 1-2 \pi^{-1}<0.363381,\left|g_{2}\right| \leq 3 / 2 \text {. }
$$

Because the nonzero coefficients in the power series (3.22) for $f(x)$ are positive, we infer from (2.8) and (2.9) that $\left|g_{1}\right| \leq f(x) \leq f(\pi / 2)=1-2 \pi^{-1},\left|g_{2}\right| \leq(1 / 2)+\varphi(x)+f^{\prime}(x) \leq(1 / 2)+$ $\varphi(\pi / 2)+f^{\prime}(\pi / 2)=3 / 2$. It is helpful to note that

$$
\varphi(x)+f^{\prime}(x)=(1+\cos x)^{-1} .
$$

Lemma 13: It is true when $0 \leq x \leq \pi / 2$ and $n \geq 2$ that

$$
\left|(2 n+1)^{-1} g_{1} / g_{0}\right|+\left|(2 n+1)^{-2} g_{2} / g_{0}\right|<0.641832 \text {. }
$$

Therefore, Lemma 2 is true when $n_{0}=2$.

The lemma is an immediate consequence of Lemmas 11 and 12 , and the fact that (2.17) is implied by (5.6). (Although adequate, this result is rather crude. It is possible to show, with the help of numerical calculations similar to those we will use below in the proofs of Lemmas 14 and 15, that the left hand side of (5.6) does not exceed 0.299394.)

The analysis to show that (2.22) is true when $n \geq 2$ and $0 \leq x \leq \pi / 2$, so that Lemma 4 is true when $n_{0}=2$, is somewhat more recondite. We begin with the following assertion.

Lemma 14: The left hand side of (2.22) does not exceed 0.884786 when $0<x \leq \pi / 2, n \geq 2$ and $z=(2 n+1) x \geq 6 \pi$.

This lemma will be a consequence of the inequalities,

$$
\begin{gathered}
f_{0} \geq 0.105264,\left|f_{1}\right| \leq 0.128401,\left|f_{2}\right| \leq 1.20476,\left|f_{3}\right| \leq 1.08086 \\
\left|f_{4}\right| \leq 5.57529,\left|f_{5}\right| \leq 4.51528,\left|f_{6}\right| \leq 3.95661
\end{gathered}
$$

when $n \geq 2$. We now proceed to prove these inequalities when $0 \leq x \leq \pi / 2$ and $z \geq 6 \pi$.

If we write (3.4) in the form,

$$
\begin{aligned}
& 8 f_{0}=1-2 z^{-1}\left(1-16 z^{-2}\right) \sin z-10 z^{-2}(1+\cos z) \\
& -8 z^{-4}\left(1-2 z^{-2}\right)(1-\cos z)^{2}-32 z^{-5}(1-\cos z) \sin z
\end{aligned}
$$

and observe that $1-16 z^{-2}$. and $1-2 z^{-2}$ are positive (when $z>4$ ), that $\sin z \leq 1$ and $-1 \leq \cos z \leq 1$, and that $(1-\cos z) \sin z \leq 3^{3 / 2} / 4$, we see that

$$
8 f_{0} \geq 1-2 z^{-1}\left(1-16 z^{-2}\right)-20 z^{-2}-32 z^{-4}\left(1-2 z^{-2}\right)-24 \cdot 3^{1 / 2} z^{-5} .
$$

The right hand side of (5.10) is an increasing function of $z$ when $z \geq 6 \pi$, because its derivative 


$$
2 z^{-2}\left(1-48 z^{-2}\right)+40 z^{-3}+128 z^{-5}\left(1-3 z^{-2}\right)+120 \cdot 3^{1 / 2} z^{-6}
$$

is positive (when $z^{2}>48$ ). If we replace $z$ by $6 \pi$ in the right hand side of (5.10), we conclude that the first inequality in (5.7) is true when $z \geq 6 \pi$.

If we write (3.14) in the form

$$
\begin{gathered}
-4 f_{1}=\left[\left(1-4 z^{-2}\right) \sin z+8 z^{-1}\left\{1-z^{-2}(1-\cos z)(3+\cos z)\right\}\right. \\
\left.+24 z^{-4}(1-\cos z) \sin z\right] f(x)
\end{gathered}
$$

and observe that $0 \leq(1-\cos z)(3+\cos z) \leq 4$, that $1-4 z^{-2}>0$ (when $z>2$ ), and that $0 \leq$ $f(x) \leq f(\pi / 2)=1-2 \pi^{-1}$, we see that

$$
4\left|f_{1}\right| \leq\left(1-4 z^{-2}+8 z^{-1}+18 \cdot 3^{1 / 2} z^{-4}\right)\left(1-2 \pi^{-1}\right) \text {. }
$$

The right hand side of (5.12) is a decreasing function of $z$ when $z \geq 6 \pi$, because its derivative,

$$
\left\{-8 z^{-2}\left(1-z^{-1}\right)-72 \cdot 3^{1 / 2} z^{-5}\right\}\left(1-2 \pi^{-1}\right)
$$

is negative (when $z>1$ ). If we replace $z$ by $6 \pi$ in the right hand side of (5.12), we conclude that the second inequality in (5.7) is true when $z \geq 6 \pi$.

With the help of (2.8), (2.10), and (2.12) we can write (3.29) in the form,

$$
24 f_{2}=6\left\{f^{\prime}(x)-f^{\prime}(0)\right\} P_{1}(z)-24 f^{2}(x)-24 \varphi(x) k_{0}(z)-P_{2}(z),
$$

in which

$$
\begin{gathered}
P_{1}(z)=5 \cos z+4 z^{-2}\left(8-3 \cos z-2 \cos ^{2} z\right) \\
-8 z^{-3}(4-\cos z) \sin z+24(1-\cos z) \cos z \\
P_{2}(z)=9-5 \cos z-4 z^{-2}\left(11+3 \cos z-2 \cos ^{2} z\right) \\
+8 z^{-3}(13-\cos z) \sin z-24 z^{-4}(1-\cos z)(3+\cos z) .
\end{gathered}
$$

Because $3 \leq 8-3 \cos z-2 \cos ^{2} z \leq 73 / 8, \quad|(4-\cos z) \sin z| \leq 4.11667$, and $|(1-\cos z) \cos z| \leq 2$, we see that

$$
\left|P_{1}(z)\right|<5+36.5 z^{-2}+32.9334 z^{-3}+48 z^{-4}<5.10803
$$

when $z \geq 6 \pi$. Moreover, it follows from (2.12) and (2.21) that

$$
\begin{gathered}
0<k_{0}(z)=\left\{1-4 z^{-1}\left(1-6 z^{-2}\right) \sin z-12 z^{-2}\left(1-2 z^{-2}\right) \cos z-24 z^{-4}\right\} / 4 \\
\leq\left\{1+4 z^{-1}\left(1-6 z^{-2}\right)+12 z^{-2}\left(1-2 z^{-2}\right)-24 z^{-4}\right\} / 4 \\
<0.310505 \text { when } z \geq 6 \pi .
\end{gathered}
$$

Because $4 \leq 9-5 \cos z \leq 14,6 \leq 11+3 \cos z-2 \cos ^{2} z \leq 97 / 8,|(13-\cos z) \sin z| \leq 13.0382$, and $0 \leq(1-\cos z)(3+\cos z) \leq 4$, we see that, when $z \geq 6 \pi$, 


$$
\begin{gathered}
P_{2}(z) \leq 14-24 z^{-2}+104.306 z^{-3}<14 \\
P_{2}(z) \geq 4-48.5 z^{-2}-104.306 z^{-3}-96 z^{-4}>3.84716 .
\end{gathered}
$$

Hence

$$
\left|P_{2}(z)\right|<14
$$

when $z \geq 6 \pi$. It now follows from (5.13), (5.16), (5.17) and (5.20) that

$$
24\left|f_{2}\right| \leq 5.10803\left\{6 f^{\prime}(\pi / 2)-1\right\}+24 f^{2}(\pi / 2)+7.452124 \varphi(\pi / 2)+14 .
$$

If we observe that $f^{\prime}(\pi / 2)=4 \pi^{-2}, f(\pi / 2)=1-2 \pi^{-1}$, and $\varphi(\pi / 2)=1-4 \pi^{-2}$, the third inequality in (5.7) is a consequence of (5.21).

It follows from (3.42), (2.8) and (2.10) that

in which

$$
f_{3}=f^{\prime \prime}(x) P_{3}(z)-2 \varphi^{\prime}(x) h_{0}(z)+f(x) \varphi(x) \sin z
$$

$$
\begin{gathered}
P_{3}(z)=\left\{1.5-z^{-2}(3+\cos z)\right\} \sin z+z^{-1}\left(3-\cos ^{2} z\right) \\
+4 z^{-3}(1-\cos z) \cos z
\end{gathered}
$$

Because $0<1.5-4 z^{-2}<1.5-z^{-2}(3+\cos z)<1.5 \quad$ (when $\left.z^{2}>8 / 3\right), \quad\left|3-\cos ^{2} z\right| \leq 3$, and $|(1-\cos z) \cos z| \leq 2$, we see that

$$
P_{3}(z) \mid \leq 1.5+3 z^{-1}+8 z^{-3}<1.66035
$$

when $z \geq 6 \pi$. Moreover, we infer from (2.10) that

$$
\left|h_{0}(z)\right| \leq z^{-1}+2 z^{-2}+4 z^{-3}<0.059278
$$

when $z \geq 6 \pi$. It now follows from (5.22), (5.24) and (5.25) that

$$
\left|f_{3}\right| \leq 1.66035 f^{\prime \prime}(\pi / 2)+0.118556 \varphi^{\prime}(\pi / 2)+f(\pi / 2) \varphi(\pi / 2) .
$$

Because $f^{\prime \prime}(\pi / 2)=1-16 \pi^{-3}, \varphi^{\prime}(\pi / 2)=16 \pi^{-3}, f(\pi / 2)=1-2 \pi^{-1}$ and $\varphi(\pi / 2)=1-4 \pi^{-2}$, we conclude that the fourth inequality in (5.7) is true when $z \geq 6 \pi$.

It follows from (2.19) that

$$
\left|f_{4}\right| \leq\left|g_{0} k_{4}\right|+\left|g_{1} k_{3}\right|+\left|g_{2} k_{2}\right|+2\left|h_{1} h_{3}\right|+h_{2}^{2} .
$$

We infer from (2.16) and (2.8) that

$$
\left|g_{0}\right|=g_{0} \leq\left(\frac{1}{2}\right)+z^{-1}<0.553052
$$

when $z \geq 6 \pi$, and from $(2.14)$ that

$$
k_{4}=f^{\prime \prime \prime}(x)(1-\cos z)-\psi(x),
$$

in which 


$$
\psi(x)=\varphi^{\prime \prime}(x)+f^{\prime \prime \prime}(x)-\left(\frac{1}{4}\right)
$$

is an increasing function for which $\psi(0)=0, \psi(\pi / 2)=7 / 4$. Hence

$$
\begin{gathered}
k_{4} \leq f^{\prime \prime \prime}(x)(1-\cos z) \leq 2 f^{\prime \prime \prime}(\pi / 2)=192 \pi^{-4}<1.97107 \\
k_{4} \geq-\psi(x) \geq-7 / 4=-1.75
\end{gathered}
$$

and we deduce from (5.28) that

$$
\left|g_{0} k_{4}\right|<1.09011 \text {. }
$$

We infer from (2.13) that $\left|k_{3}\right| \leq 3 f^{\prime \prime}(\pi / 2)=3\left(1-16 \pi^{-3}\right)<1.45193$, so that an appeal to Lemma 12 shows that

$$
\left|g_{1} k_{3}\right| \leq 0.527604 \text {. }
$$

Similarly, it follows from (2.13) and Lemma 12 that

$$
\left|g_{2} k_{2}\right|<\left(\frac{3}{2}\right)\left\{\left(\frac{1}{2}\right)+3 f^{\prime}\left(\frac{\pi}{2}\right)\right\}=\left(\frac{3}{4}\right)+\left(\frac{18}{\pi^{2}}\right)<2.57379,
$$

and from (2.11) and (5.5) that

$$
\begin{gathered}
2\left|h_{1} h_{3}\right| \leq 2 f(x)\left\{\varphi^{\prime}(x)+f^{\prime \prime}(x)\right\} \leq 2 f\left(\frac{\pi}{2}\right)<0.726762 \\
h_{2}^{2} \leq 4 f^{\prime 2}(x) \leq 4 f^{\prime 2}\left(\frac{\pi}{2}\right)=64 \pi^{-4}<0.657023 .
\end{gathered}
$$

If we use (5.24) and (5.31) through (5.35), we conclude that the first inequality in (5.8) is true when $z \geq 6 \pi$.

It follow from (2.19) and some results from the previous paragraph that

$$
\begin{gathered}
\left|f_{5}\right| \leq\left|g_{1} k_{4}\right|+\left|g_{2} k_{3}\right|+2\left|h_{2} h_{3}\right| \\
\leq 192 \pi^{-4}\left(1-2 \pi^{-1}\right)+\left(\frac{9}{2}\right)\left(1-16 \pi^{-3}\right)+16 \pi^{-2} .
\end{gathered}
$$

Therefore, the second inequality in (5.8) is true. Similarly,

$$
\left|f_{6}\right| \leq\left|g_{2} k_{4}\right|+h_{3}^{2} \leq 288 \pi^{-4}+1
$$

so that the third inequality of (5.8) is true. The proof of Lemma 14 is now complete.

Lemma 15: The left hand side of (2.22) does not exceed 0.919051 when $0 \leq x \leq \pi / 2, n \geq 2$ and $z=(2 n+1) x \leq 6 \pi$.

We define $\xi$ so that

$$
\xi=\min \left(\frac{z}{5}, \frac{\pi}{2}\right)
$$

It is clear that $0 \leq x \leq \xi$ when $n \geq 2$. It then follows from (3.14) that

$$
\left|f_{1}\right| \leq P_{0}(z) f(\xi)
$$

in which 


$$
\begin{gathered}
P_{0}(z)=\mid 4^{-1} \sin z+2 z^{-1}-z^{-2} \sin z-2 z^{-3}(1-\cos z)(3+\cos z) \\
+6 z^{-4}(1-\cos z) \sin z \mid .
\end{gathered}
$$

Similarly, it follows from (5.13) and (5.22) that

$$
\begin{gathered}
\left|f_{2}\right| \leq 24^{-1}\left[\left\{6 f^{\prime}(\xi)-1\right\}\left|P_{1}(z)\right|+\left|P_{2}(z)\right|\right]+f^{2}(\xi)+\varphi(\xi) k_{0}(z), \\
\left|f_{3}\right| \leq f^{\prime \prime}(\xi)\left|P_{3}(z)\right|+2 \varphi^{\prime}(\xi)\left|h_{0}(z)\right|+f(\xi) \varphi(\xi)|\sin z| .
\end{gathered}
$$

(We have used the implication of $(2.21)$ that $k_{0}(z) \geq 0$.)

The analysis for $f_{4}$ is more elaborate. We first deduce from (5.29) that

$$
\left\{f^{\prime \prime \prime}(0)(1-\cos z)-\psi(\xi)\right\} g_{0}(z) \leq g_{0} k_{4} \leq f^{\prime \prime \prime}(\xi)(1-\cos z) g_{0}(z),
$$

and from (2.8) and (2.31) that

$$
0 \leq g_{1} k_{3}=3 f(x) f^{\prime \prime}(x) \sin ^{2} z \leq 3 f(\xi) f^{\prime \prime}(\xi) \sin ^{2} z .
$$

We next use (2.9) and (2.13) to see that

$$
\begin{gathered}
g_{2} k_{2}=\left\{\left(\frac{1}{2}\right)+\varphi(x)+f^{\prime}(x)\right\}\left\{\left(\frac{1}{2}\right)-3 f^{\prime}(x)\right\} \\
+\left\{1+3 \varphi(x)+6 f^{\prime}(x)\right\} f^{\prime}(x)(1-\cos z)-3 f^{\prime 2}(x)(1-\cos z)^{2},
\end{gathered}
$$

so that

$$
\begin{gathered}
\left\{\left(\frac{1}{2}\right)+\varphi(\xi)+f^{\prime}(\xi)\right\}\left\{\left(\frac{1}{2}\right)-3 f^{\prime}(\xi)\right\}+\left\{1+3 \varphi(0)+6 f^{\prime}(0)\right\} f^{\prime}(0)(1-\cos z) \\
-3 f^{\prime 2}(\xi)(1-\cos z)^{2} \leq g_{2} k_{2} \leq\left\{1+3 \varphi(\xi)+6 f^{\prime}(\xi)\right\} f^{\prime}(\xi)(1-\cos z) \\
-3 f^{\prime 2}(0)(1-\cos z)^{2}
\end{gathered}
$$

We now use (2.11) to see that

$$
-2 h_{1} h_{3}=2\left\{\varphi^{\prime}(x)+f^{\prime \prime}(x) \cos z\right\} f(x) \cos z \leq 2\left\{\varphi^{\prime}(\xi)+f^{\prime \prime}(\xi)\right\} f(\xi) .
$$

We observe that $-2 h_{1} h_{3}$ is a quadratic function of $\cos z$ whose absolute minimum is $-\varphi^{\prime 2}(x) f(x) / 2 f^{\prime \prime}(x)$. We have tabulated the function $\varphi^{\prime 2}(x) f(x) / f^{\prime \prime}(x)$ when $x=0(\pi / 36) \pi / 2$, and have observed that it is an increasing function of $x$. Therefore,

$$
-\varphi^{\prime 2}(\xi) f(\xi) / 2 f^{\prime \prime}(\xi) \leq-2 h_{1} h_{3} \leq 2\left\{\varphi^{\prime}(\xi)+f^{\prime \prime}(\xi)\right\} f(\xi) .
$$

Finally, we use (2.11) to see that

$$
-4 f^{\prime 2}(\xi) \sin ^{2} z \leq-h_{2}^{2}=-4 f^{\prime 2}(x) \sin ^{2} z \leq-4 f^{\prime 2}(0) \sin ^{2} z .
$$

It now follows from the definition of (2.19) of $f_{4}$, the inequalities (5.43) through (5.47), and the numerical values, $f^{\prime}(0)=1 / 6, f^{\prime \prime \prime}(0)=7 / 60, \varphi(0)=1 / 3$, obtained from (3.22), that

$$
\left|f_{4}\right| \leq \max \left\{\left|P_{4}(z)\right|,\left|P_{5}(z)\right|\right\},
$$


in which

$$
\begin{gathered}
P_{4}(z)=\left[f^{\prime \prime \prime}(\xi) g_{0}(z)+\left\{1+3 \varphi(\xi)+6 f^{\prime}(\xi)\right\} f^{\prime}(\xi)\right. \\
\left.-12^{-1}(1-\cos z)\right](1-\cos z)+\left\{3 f(\xi) f^{\prime \prime}(\xi)-\left(\frac{1}{9}\right)\right\} \sin ^{2} z \\
+2\left\{\varphi^{\prime}(\xi)+f^{\prime \prime}(\xi)\right\} f(\xi), \\
P_{5}(z)=\left\{\left(\frac{7}{60}\right)(1-\cos z)-\psi(\xi)\right\} g_{0}(z)+\left\{\left(\frac{1}{2}\right)+\varphi(\xi)\right. \\
\left.+f^{\prime}(\xi)\right\}\left\{\left(\frac{1}{2}\right)-3 f^{\prime}(\xi)\right\}+2^{-1}(1-\cos z)-3 f^{\prime 2}(\xi)(1-\cos z)^{2} \\
-\varphi^{\prime 2}(\xi) f(\xi) / 2 f^{\prime \prime}(\xi)-4{f^{\prime}}^{2}(\xi) \sin ^{2} z .
\end{gathered}
$$

It follows from (2.19), (2.9), (2.11), (2.13) and (2.14) that

$$
f_{5}=\left\{P_{6}(x) \cos z+P_{7}(x)\right\} \sin z
$$

in which

$$
\begin{gathered}
P_{6}(x)=f(x) f^{\prime \prime \prime}(x)-f^{\prime}(x) f^{\prime \prime}(x) \\
P_{7}(x)=f(x)\left\{\varphi^{\prime \prime}(x)-\left(\frac{1}{4}\right)\right\}-4 f^{\prime}(x) \varphi^{\prime}(x)+3 f^{\prime \prime}(x)\left\{\varphi(x)+\left(\frac{1}{2}\right)\right\} .
\end{gathered}
$$

We have tabulated the functions $P_{6}(x)$ and $P_{7}(x)-P_{6}(x)$ when $x=0(\pi / 36) \pi / 2$, and have observed that each of them is a positive increasing function of $x$ when $0<x \leq \pi / 2$. We conclude that

$$
\left|f_{5}\right| \leq\left\{P_{7}(\xi)+P_{6}(\xi) \cos z\right\}|\sin z|
$$

It follows from (2.19), (2.9), (2.11) and (2.14) that

$$
f_{6}=P_{8}(x)+P_{9}(x) \cos z+P_{10}(x) \cos ^{2} z,
$$

in which

$$
\begin{gathered}
P_{8}(x)=-\left\{\left(\frac{1}{2}\right)+\varphi(x)\right\}\left\{\left(\frac{1}{4}\right)-\varphi^{\prime \prime}(x)\right\}-\varphi^{\prime 2}(x), \\
P_{9}(x)=-\left\{\left(\frac{1}{4}\right)-\varphi^{\prime \prime}(x)\right\} f^{\prime}(x)+\left\{\left(\frac{1}{2}\right)+\varphi(x)\right\} f^{\prime \prime \prime}(x)-2 \varphi^{\prime}(x) f^{\prime \prime}(x), \\
P_{10}(x)=f^{\prime}(x) f^{\prime \prime \prime}(x)-f^{\prime \prime 2}(x) .
\end{gathered}
$$

We rewrite (5.55) in the form

$$
f_{6}=\left(P_{8}+P_{9}+P_{10}\right)-\left(P_{9}+2 P_{10}\right)(1-\cos z)+P_{10}(1-\cos z)^{2}
$$

and observe, after tabulating $P_{9}(x)$ and $P_{10}(x)$ when $x=0(\pi / 36) \pi / 2$, that each of them is a positive increasing function of $x$ when $0 \leq x \leq \pi / 2$. Moreover,

$$
8\left\{P_{8}(x)+P_{9}(x)+P_{10}(x)\right\}=\left(13-3 \cos x-9 \cos ^{2} x-\cos ^{3} x\right) /(1+\cos x)^{3},
$$

so that $P_{8}(x)+P_{9}(x)+P_{10}(x)$ is also a positive increasing function of $x$ when $0<x \leq \pi / 2$. We 
conclude that

$$
-\left\{P_{9}(x)+2 P_{10}(x)\right\}(1-\cos z) \leq f_{6} \leq P_{8}(x)+P_{9}(x)+P_{10}(x)
$$

so that

$$
\left|f_{6}\right| \leq \max \left[P_{8}(\xi)+P_{9}(\xi)+P_{10}(\xi),\left\{P_{9}(\xi)+2 P_{10}(\xi)\right\}(1-\cos z)\right]
$$

If we let $f_{r}^{*}(z) \quad(r=1,2, \ldots, 6)$ be the right hand sides of the inequalities (5.39), (5.41), (5.42), (5.48), (5.54) and (5.62), respectively, then the left hand side of (2.22) does not exceed

$$
\theta(z)=f_{0}^{-1}(z) \sum_{r=1}^{6} 5^{-r} f_{r}^{*}(z)
$$

when $n \geq 2$. We have tabulated $\theta(z)$ when $z=0(\pi / 18) 6 \pi$, and used these results to search, by appropriate subtabulation, for $\max _{0} \leq z \leq 6 \pi \theta(z)$. In this way, we find that $\theta(z)<0.919051$. This completes the proof of Lemma 15.

Lemma 16: The inequality (2.22) is true when $n \geq 2$ and $0 \leq x \leq \pi / 2$. Therefore, Lemmas 4,5 and 6 are also true when $n_{0}=2$.

The first sentence in Lemma 16 is an immediate consequence of Lemmas 14 and 15 . Therefore, Lemma 4 is true when $n_{0}=2$. This remark and Lemma 13 then show that Lemmas 5 and 6 are true when $n_{0}=2$.

\section{References}

[1] Abramowitz, M. and Stegun, I.A. (eds.), Handbook of Mathematical Functions, with Formulas, Graphs and Mathematical Tables, 10th printing, John Wiley, New York 1972.

[2] Bharucha-Reid, A.T. and Sambandham, M., Random Polynomials, Academic Press, Orlando, Florida 1986.

[3] Cramer, H. and Leadbetter, M.R., Stationary and Related Stochastic Processes, John Wiley, New York 1967.

[4] Das, M., The average number of real zeros of a random trigonometric polynomial, Proc. Camb. Phil. Soc., 64 (1968), 721-729.

[5] Davis, H.T., The Summation of Series, Principia Press of Trinity University, San Antonio, Texas 1962.

[6] Wilkins, J.E., Mean number of real zeros of a random trigonometric polynomial, Proc. AMS, 111 (1991), 851-863.

[7] Wilkins, J.E., Mean number of real zeros of a random trigonometric polynomial. II, In: Topics in Polynomials of One and Several Variables, (ed. by Th. M. Rassias, H.M. Srivastava and A. Yanushauskas), World Scientific Publishing Co., Singapore (1993), 581594 . 


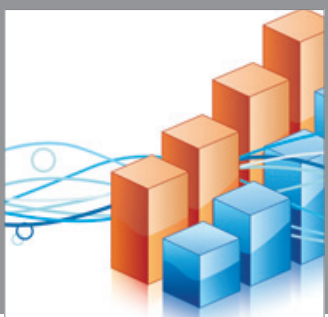

Advances in

Operations Research

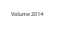

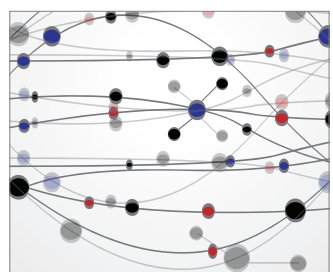

\section{The Scientific} World Journal
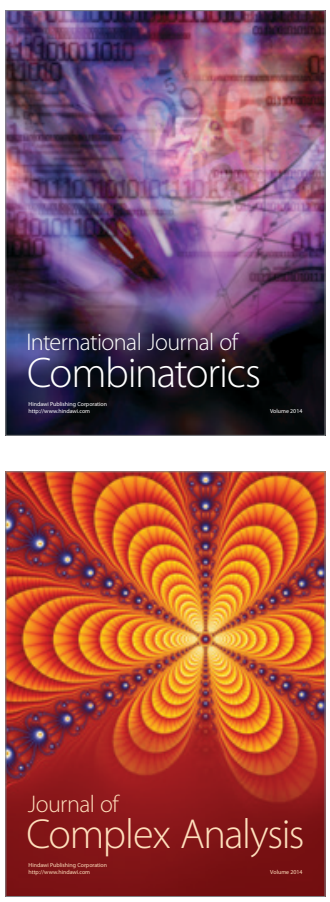

International Journal of

Mathematics and

Mathematical

Sciences
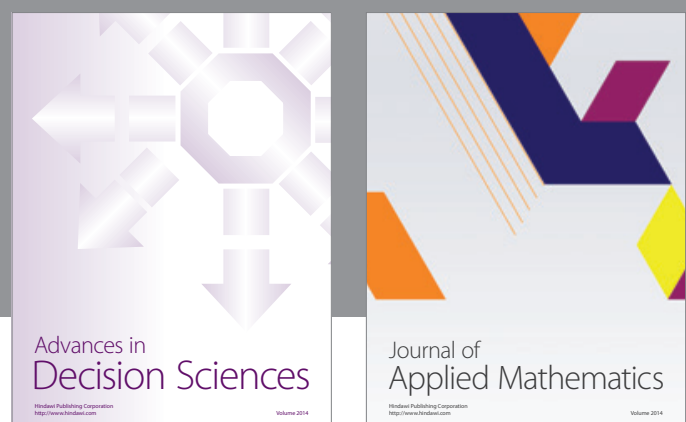

Journal of

Applied Mathematics
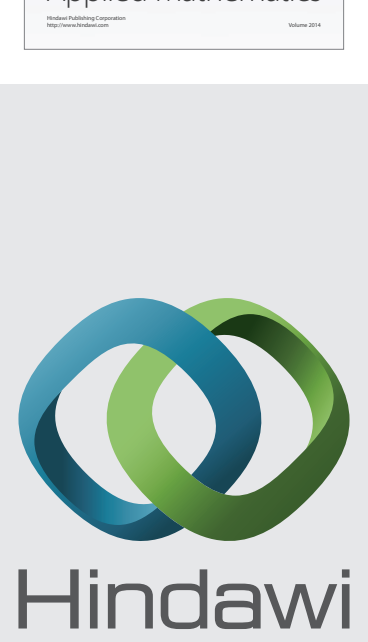

Submit your manuscripts at http://www.hindawi.com
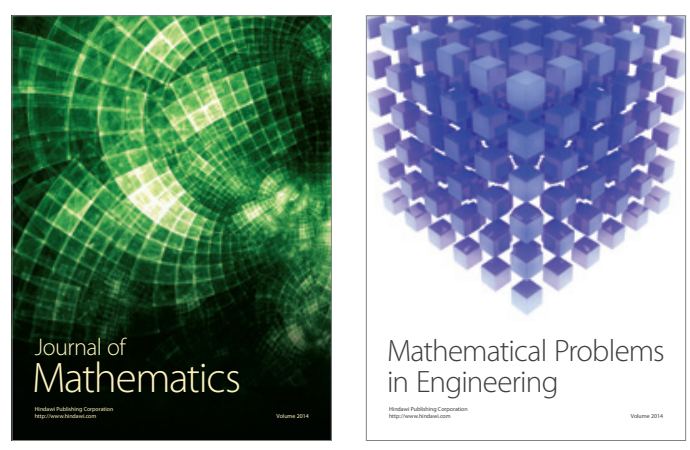

Mathematical Problems in Engineering
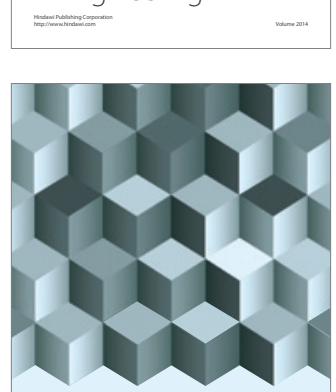

Journal of

Function Spaces
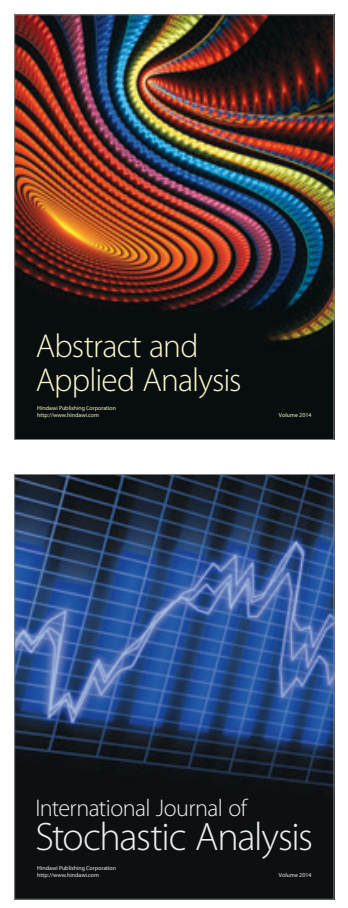

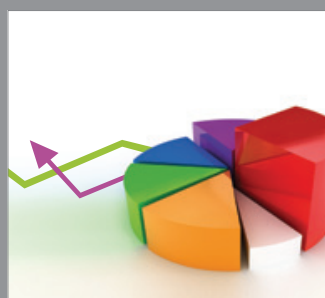

ournal of

Probability and Statistics

Promensencen
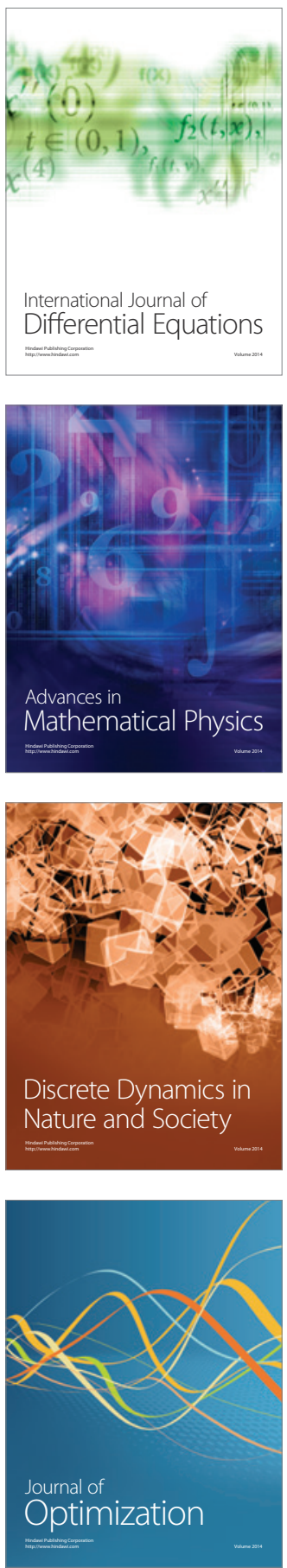